\title{
Análise bibliométrica da produção científica internacional das universidades, em torno dos Objetivos de Desenvolvimento Sustentável (ODS), representada na Web of
}

\section{Science (WoS)}

\author{
Bibliometric analysis of the international scientific production of universities around the
}

Sustainable Development Goals (SDGs), represented in the Web of Science (WoS)

Análisis bibliométrico de la producción científica internacional de las universidades en torno a los Objetivos de Desarrollo Sostenible (ODS), representados en la Web of Science (WoS)

\section{Resumo}

As universidades, como promotoras do conhecimento e do desenvolvimento social, têm levado à inclusão dos ODS nos seus programas e agendas internas, por meio das práticas do ensino, da pesquisa e da extensão. Nesse sentido, esta pesquisa tem como objetivo analisar a produção científica sobre os Objetivos de Desenvolvimento Sustentável (ODS) desenvolvida nas universidades, em diferentes países, constante na base de dados Web of Science, no período de 2016 a 2020. Para tanto, realizou-se uma pesquisa exploratória-descritiva, utilizando-se o software VosViewer, em seguida, fez-se uma análise das redes bibliométricas que caracterizam a produção internacional acerca do tema. Com enfoque qualitativo, estudou-se o conteúdo das obras de maior impacto em torno dessa temática, cujos resultados apontam para formação de clusters de autores, organizações e países. Os termos mais destacados giram em torno das palavras-chave definidas como foco do estudo na pesquisa inicial. A publicação com maior score de citações foi: Sustainable Development Goals and sustainability teaching at universities: Falling behind or getting ahead of the pack?, de Leal Filho, W. (2019).

Palavras-chave: Objetivos de desenvolvimento sustentável; Bibliometria; Universidades.

\begin{abstract}
Universities, as promoters of knowledge and social development, have led to the inclusion of the SDGs in their programs and internal agendas, through teaching, research and extension practices. In this sense, this research aims to analyze the scientific production on the Sustainable Development Goals (SDGs) developed in universities, in different countries, included in the Web of Science database, between 2016 and 2020. To this end, exploratory-descriptive research was carried out, using the VosViewer software, then an analysis was made of the bibliometric networks that characterize international production on the subject. With a qualitative focus, the content of the works with the greatest impact around this theme was studied, the results of which point to the formation of clusters of authors, organizations and countries. The most prominent terms revolve around the keywords defined as the focus of the study in the initial research. The publication with the highest citation score was: Sustainable Development Goals and sustainability teaching at universities: Falling behind or getting ahead of the pack?, by Leal Filho, W. (2019).
\end{abstract}

Keywords: Sustainable development goals; Bibliometric; Universities.

\section{Resumen}

Las universidades, como promotoras del conocimiento y el desarrollo social, han propiciado la inclusión de los ODS en sus programas y agendas internas, a través de prácticas de docencia, investigación y extensión. En este sentido, esta investigación tiene como objetivo analizar la producción científica sobre los Objetivos de Desarrollo Sostenible (ODS) desarrollada en universidades, en diferentes países, incluidas en la base de datos Web of Science, en el período de 2016 a 2020. Con este fin, la investigación exploratoria-descriptiva se llevó a cabo utilizando el software VosViewer, a continuación, se hizo un análisis de las redes bibliométricas que caracterizan la producción internacional en la materia. Con un enfoque cualitativo, se estudió el contenido de los trabajos con mayor impacto en 
esta temática, cuyos resultados apuntan a la formación de clusters de autores, organizaciones y países. Los términos más destacados giran en torno a las palabras clave definidas como el foco del estudio en la investigación inicial. La publicación con la puntuación más alta en citas fue: Sustainable Development Goals and sustainability teaching at universities: Falling behind or getting ahead of the pack?, de Leal Filho, W. (2019).

Palabras clave: Objetivos de desarrollo sostenible; Bibliometría; Universidades.

\section{Introdução}

A discussão em torno dos Objetivos de Desenvolvimento Sustentável (ODS) surgiu em 2012, durante a Conferência sobre Desenvolvimento Sustentável no Rio de Janeiro. A ideia era produzir um conjunto de metas universais que suprisse os desafios ambientais, políticos e econômicos mais urgentes que nosso mundo enfrenta. Nesse contexto, a ONU criou, em 2015, os 17 Objetivos de Desenvolvimento Sustentável que visam acabar com a pobreza, fome e desigualdade; realizar mudanças climáticas e ações ambientais; melhorar acesso à saúde e educação; e contribuir para instituições e parcerias fortes, entre outros, todos até 2030.

Após mais de dois anos de negociações, em 25 de setembro de 2015, chefes de Estado e altos representantes dos 193 países-membros integrantes da Assembleia Geral da ONU adotaram o documento intitulado "Transformando Nosso Mundo: A Agenda 2030 para o Desenvolvimento Sustentável”, um plano de ação para as pessoas, o planeta e a prosperidade. No documento, os países-membros da ONU reconhecem que "a erradicação da pobreza em todas as suas formas e dimensões, incluindo a pobreza extrema, é o maior desafio global e um requisito indispensável para o desenvolvimento sustentável”. Um dos compromissos assumidos na Agenda é o de "não deixar ninguém para trás", em referência aos mais pobres.

As metas dos Objetivos de Desenvolvimento Sustentável, voltadas à qualidade da educação, realçam a responsabilidade que as instituições de ensino superior podem ter no alcance dos ODS (UNESCO, 2017). A partir desta pesquisa, pretende-se conhecer como os estudos sobre a implementação dos ODS vêm sendo inseridos no ensino superior, e como as instituições estão se preparando para responder ao desafio lançado pelas Nações Unidas (2015) para a promoção do desenvolvimento sustentável.

Nesse sentido, reconhece-se que as Universidades, como responsáveis pelo desenvolvimento social, estão diante de um grande desafio no sentido de incluir os ODS em seus em seus programas e agendas internas. É necessário compreender, no entanto, que o atingimento das metas e objetivos pactuados no âmbito dos ODS, exige um esforço coordenado não apenas no nível das esferas governamentais, mas também da iniciativa privada, das ONGs e de toda a sociedade brasileira.

Embora os Objetivos de Desenvolvimento Sustentável tenham uma abordagem global, as ações desenvolvidas, por cada país, são locais e dependem do estágio em que cada um se encontra para atingir esses objetivos. Além disso, o grau de desenvolvimento e compromisso com a sustentabilidade de cada país afeta seus interesses e ações internas.

Estudos apontam que existem várias discussões teóricas recentes sobre educação para o desenvolvimento sustentável (Annan-Diabe Molinari, 2017; Dlouhá e Pospíšilová, 2018; Galli et al., 2018; Hák et al., 2016; Rede de Soluções de Desenvolvimento Sustentável, 2015; Nações Unidas, 2017a; Banco Mundial, 2017a). No entanto, há poucas publicações que mostram até que ponto os ODS estão sendo alcançados em todo o mundo e os desafios vistos nas diversas regiões geográficas.

No Brasil, a governança para implementação dos ODS foi instituída por meio do Decreto $\mathrm{n}^{\circ} 8.892$, de 27 de outubro de 2016, que criou a Comissão Nacional para os Objetivos de Desenvolvimento Sustentável (CNODS). Decorrido um ano de sua criação, em outubro de 2017 a CNODS publicou o seu Plano de Ação 2017-2019, o qual previa, como parte da estratégia da Agenda 2030 Brasil, a adequação das 169 metas dos ODS e seus respectivos indicadores globais à realidade brasileira. Assim, as tarefas de "nacionalização" das metas e indicadores foram atribuídas ao Ipea e ao IBGE, respectivamente, definidos como órgãos de assessoramento permanente da CNODS no âmbito de sua estrutura de governança.

Como se pode constatar, as metas contidas nos 17 ODS representam uma excelente oportunidade de pautar a criação e 
o aprimoramento de políticas públicas, programas, no interior das universidades e assim, fazer com que estas se tornem um indutor do país rumo ao desenvolvimento sustentável desejado.

Nesse contexto, De la Poza (2021) compreende que "os 17 ODS representam desafios para as IES abordarem essas questões globais, especificamente o ODS4, cujo objetivo é fornecer acesso universal ao ensino superior de qualidade".

Percebe-se, portanto, que o desafio maior para as universidades, no momento, é desenvolver estratégias que possibilitem a internalização dos ODS, permitindo que seu potencial de indutor do desenvolvimento, tanto local, como regional e nacional, de fato, se concretize e traga os benefícios almejados para a nossa sociedade, até 2030.

Nesse sentido, este artigo tem como objetivo analisar a produção científica desenvolvida nas Universidades, em torno dos Objetivos de Desenvolvimento Sustentável, identificando as principais publicações indexadas na Web of Science, dentro de seu acervo principal, no período de 2016 a 2020. Para tanto, examinamos os tipos de documentos publicados, a evolução do número de publicações, os países de origem das publicações, os artigos mais citados, juntamente com os autores mais produtivos e uma análise de co-autorias, com o propósito de dar visibilidade à produção científica sobre os ODS, nas Universidades, mostrando a importância e o impacto do trabalho acadêmico realizado neste sistema científico.

\section{Metodologia}

Trata-se de uma pesquisa bibliométrica que, de acordo com Araújo (2006), consiste em uma técnica quantitativa e estatística de medição dos índices de produção e disseminação de conhecimento científico vislumbrando a análise quantitativa de informações bibliográficas. A utilização desta abordagem justifica- se pelo fato de que se desenvolveu a pesquisa com o objetivo de ampliar o conhecimento referente às publicações das universidades, relacionadas à temática dos ODS, a partir do levantamento de artigos que versam sobre o assunto.

Para tanto, realizou-se uma pesquisa exploratória-descritiva, com a finalidade de mapear a produção científica internacional sobre a referida temática, utilizando-se como base de busca a Web of Science, por ser uma das mais usadas nos estudos de citação. A pesquisa foi realizada em 29 de março de 2021 e, para o delineamento da amostra, adotou-se o procedimento indicado no Quadro 1:

Quadro 1 - Procedimento para o refinamento da amostra na Web of Science (WoS).

\begin{tabular}{|c|c|c|}
\hline Consulta inicial & $1^{0}$. filtro: 115 artigos & $2^{0 \cdot}$ filtro: 88 artigos \\
\hline $\begin{array}{l}\text { Intervalo de tempo considerado: } \\
2016 \text { - } 2020 \\
\text { Índice de citações: } \\
\text { Social Sciences Citation Index } \\
\text { (SCI-EXPANDED) } \\
\text { Science Citation Index Expanded } \\
\text { (SSCI) } \\
\text { Emerging Sources Citation Index } \\
\text { (ESCI) }\end{array}$ & $\begin{array}{l}\text { Categorias da WoS: } \\
\text { Green Sustainable } \\
\text { Science } \\
\text { Technology, or } \\
\text { Education } \\
\text { Educational } \\
\text { Research, or } \\
\text { Management }\end{array}$ & $\begin{array}{l}\text { Tipos de Documento: } \\
\text { Article or Review (excluindo Early Access or Editorial Material) } \\
\text { Idioma: Inglês } \\
\text { Áreas de pesquisa: } \\
\text { Science Technology Other Topics or Engineering or Meteorology } \\
\text { Atmospheric Sciences or Environmental Sciences Ecology or Public } \\
\text { Environmental Occupational Health or Remote Sensing or Education } \\
\text { Educational Research or Imaging Science Photographic Technology or } \\
\text { Social Sciences Other Topics or Business Economics or Information } \\
\text { Science Library Science } \\
\text { Países: } \\
\text { England or Iran or Spain or South Korea or Germany or Switzerland } \\
\text { or Brazil or Taiwan or USA or Wales or Italy or Canada or Peoples R } \\
\text { China or France or Mexico or Austria or Chile or Portugal or } \\
\text { Scotland or South Africa or Estonia or India }\end{array}$ \\
\hline
\end{tabular}


Conforme indicado no quadro 1, a consulta inicial foi realizada a partir dos descritores: "Sustainable Development Goals" and "Universities", que apontaram um total de 151 artigos. Em seguida, foram realizadas 2 etapas de filtragem. Na primeira, procedeu-se com uma seleção por categorias, mantendo-se as sugeridas pela Web of Science e, na segunda, optou-se por manter artigos e revisões, excluindo-se os outros tipos de documentos que não estivessem dentro do escopo do tema em estudo, totalizando, 88 publicações, estas geraram 688 citações.

A média de citação, por item, de 7,82 e o h-index (artigos mais relevantes da amostra, da WOS) indicou um total de 12 (doze) artigos que mais impactaram dentro da temática analisada.

Posteriormente, o resultado foi exportado, gravado em um formato de arquivo separado por tabulações, objetivando-se a utilização dos dados em softwares de suporte em organização e análises bibliométricas. Para tanto, utilizou-se o software VOSviewer version 1.6.12 que oferece ferramentas para construção e visualização de redes bibliométricas (Van Eck \& Waltman, 2017).

Com base nos critérios estabelecidos para realização da pesquisa, foram criados os seguintes mapas, baseados em dados bibliográficos: tipo de análise "co-authorship" e unidade de análise "authors", "organizations" e "countries", tipo de análise "co-occurrence", com unidade de análise "all keywords" e tipo de análise "citation", com unidade de análise "documents". Tais critérios foram utilizados com o intuito de mostrar a relação de autores que publicam em co-autoria e estão definidos no quadro a seguir:

Quadro 2 - Definição dos parâmetros de corte no VOSviewer.

\begin{tabular}{|l|l|l|}
\hline \multicolumn{2}{|c|}{ PARÂMETROS } & \multicolumn{1}{c}{ DESCRIÇÃO } \\
\cline { 1 - 2 } Tipo de Análise & Unidade de Análise & \\
\cline { 1 - 2 } Co-authorship & Authors & $\begin{array}{l}\text { Relação de força que ocorre quando, em síntese, dois autores são co-citados, ou seja, } \\
\text { quando um terceiro autor os cita conjuntamente. }\end{array}$ \\
\cline { 1 - 2 } Co-authorship & Organizations & $\begin{array}{l}\text { Relação de força que ocorre quando, em síntese, autores de duas organizações são co- } \\
\text { citados, ou seja, quando um terceiro autor (de uma terceira organização) os cita } \\
\text { conjuntamente. }\end{array}$ \\
\cline { 1 - 2 } Co-authorship & Countries & $\begin{array}{l}\text { Relação de força que ocorre quando, em síntese, autores de dois países são co-citados, } \\
\text { ou seja, quando um terceiro autor (de um terceiro país) os cita conjuntamente. }\end{array}$ \\
\hline Co-occurrence & All Keywords & Relação de força e frequência entre palavras-chave. \\
\hline
\end{tabular}

Fonte: Adaptado de Van Eck et al. (2017).

Após a definição desses parâmetros, procedeu-se à análise nas obras consideradas referência, destacando o principal objetivo de cada, sua natureza e conclusões mais destacadas. Sequencialmente, realizou-se o detalhamento das principais obras identificadas na pesquisa.

\section{Resultados e Discussão}

Com base na análise realizada, é possível observar que o ano de 2016 é o período em que, efetivamente, se dá o início aos escritos de produções científicas envolvendo o tema, de modo que essa produção vai se intensificando ao longo dos anos, com um aumento substancial nos anos de 2019 e 2020, conforme se observa no gráfico a seguir: 


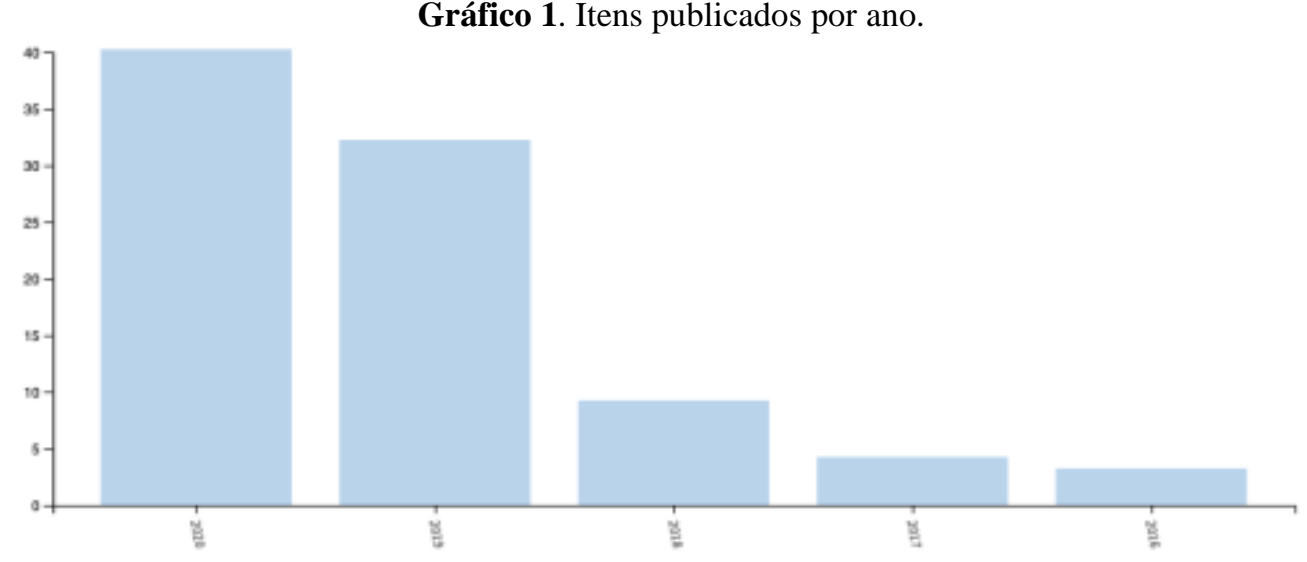

Fonte: Web of Science (2021)

Em relação à produção por co-autoria, observada nos mapas de redes, formatados a partir dos parâmetros elencados no Quadro 2 e elaborados a partir do VosViewer, foi possível realizar a análise de co-autoria e identificar como pesquisadores, instituições e países se relacionam, de acordo com os estudos que realizam e publicam conjuntamente.

A Figura 1, a seguir, apresenta a rede de co-autoria, com as características da colaboração entre os autores mais relevantes. Dada a natureza exploratória do estudo, não se trabalhou com restrição de número mínimo de trabalhos por autor.

Figura 1. Rede de co-autoria, por autores.

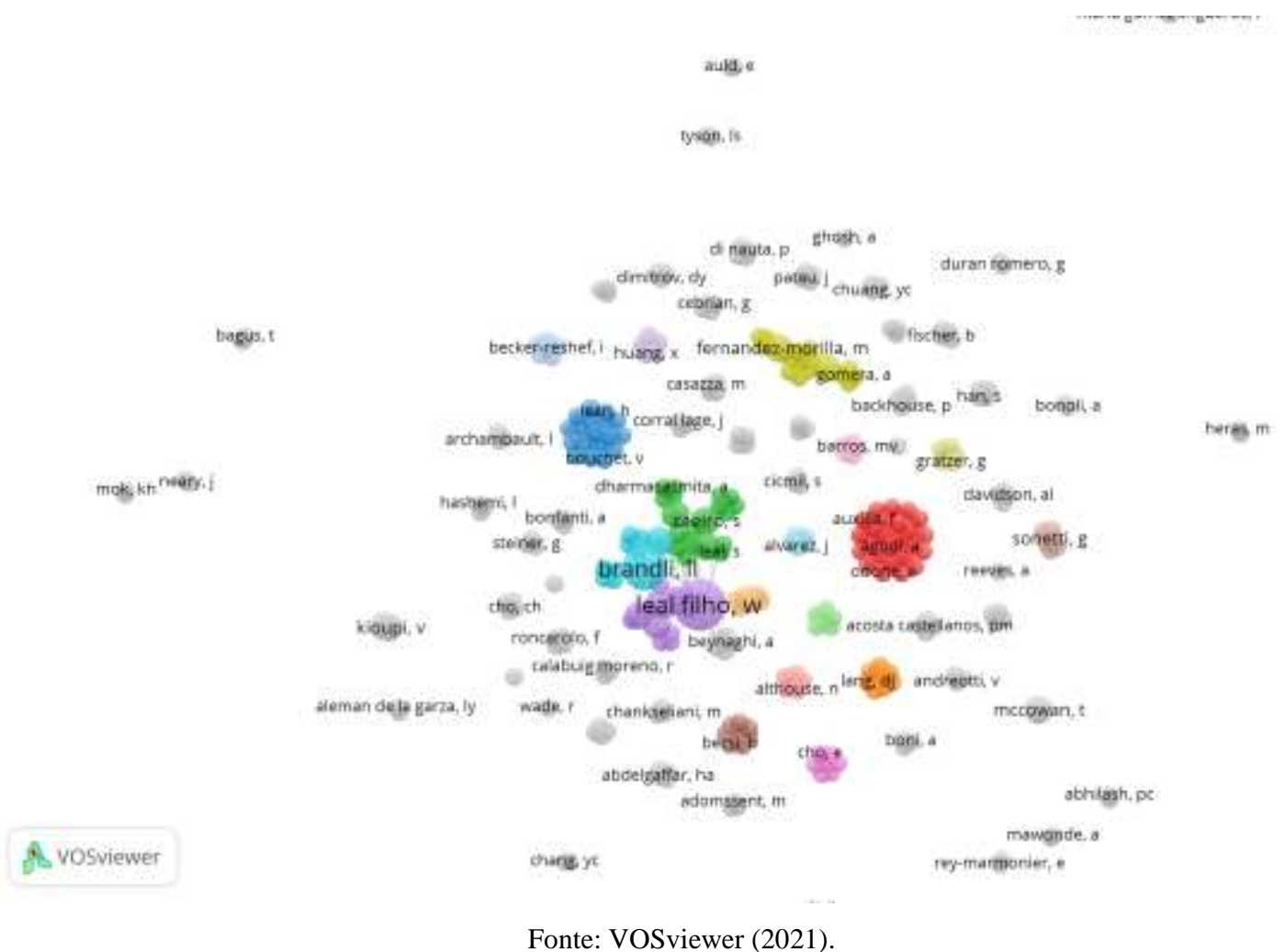

Em relação à temática em estudo, foram identificados 329 autores. Um total de 73 clusters com 1017 links entre autores. Muitos destes, conforme se observa na Figura 1, não compõem nenhum cluster ou pertencem a clusters de tamanhos reduzidos. Nesse caso, observa-se que, apenas 13 autores aparecem conectados. Isso se explica em função da 
contemporaneidade do tema em estudo, pois, embora os ODS tenham sido criados em 2015, considerou-se, para esta pesquisa, os trabalhos publicados a partir de 2016 .

O principal cluster é formado por 25 autores (em vermelho na Figura 2). Importa observar que nenhum autor nesse cluster possui mais que uma publicação e todos possuem relação de força tamanho 24 , o que garante a todos um destaque equilibrado.

O segundo cluster é composto por 19 autores (em verde na Figura 2). O destaque nesse cluster é Caeiro, S., com 2 publicações e uma relação de força tamanho 15, seguido de Mouthan-hill P., com 2 publicações e relação de força $14 \mathrm{e}$ Azeiteiro, Um., com 2 publicações e relação de força tamanho 12. O terceiro cluster de destaque é composto por 18 autores (em azul na figura 2). Novamente, nenhum autor possui mais que um trabalho publicado. Neste caso, todos os autores têm relação de força tamanho 17. Já no quarto cluster, apenas dois autores se destacam, a saber: Fernandez Morilla, M. com 2 publicações e relação de força 13 e Ruiz-Morales, J., com 2 publicações e relação de força 12.

Vale destacar no quinto cluster, o autor Leal Filho, W., que conta com 7 publicações e 7 publicações. Nos demais clusters, observa-se que a relação de força vai caindo com a diminuição dos tamanhos dos clusters, e com o número de publicações.

A Figura 2 mostra a rede de co-autoria por organizações. Assim como foi o procedimento padrão para a geração dos mapas, não houve corte por número mínimo de citações.

Figura 2. Rede de co-autoria por organizações.

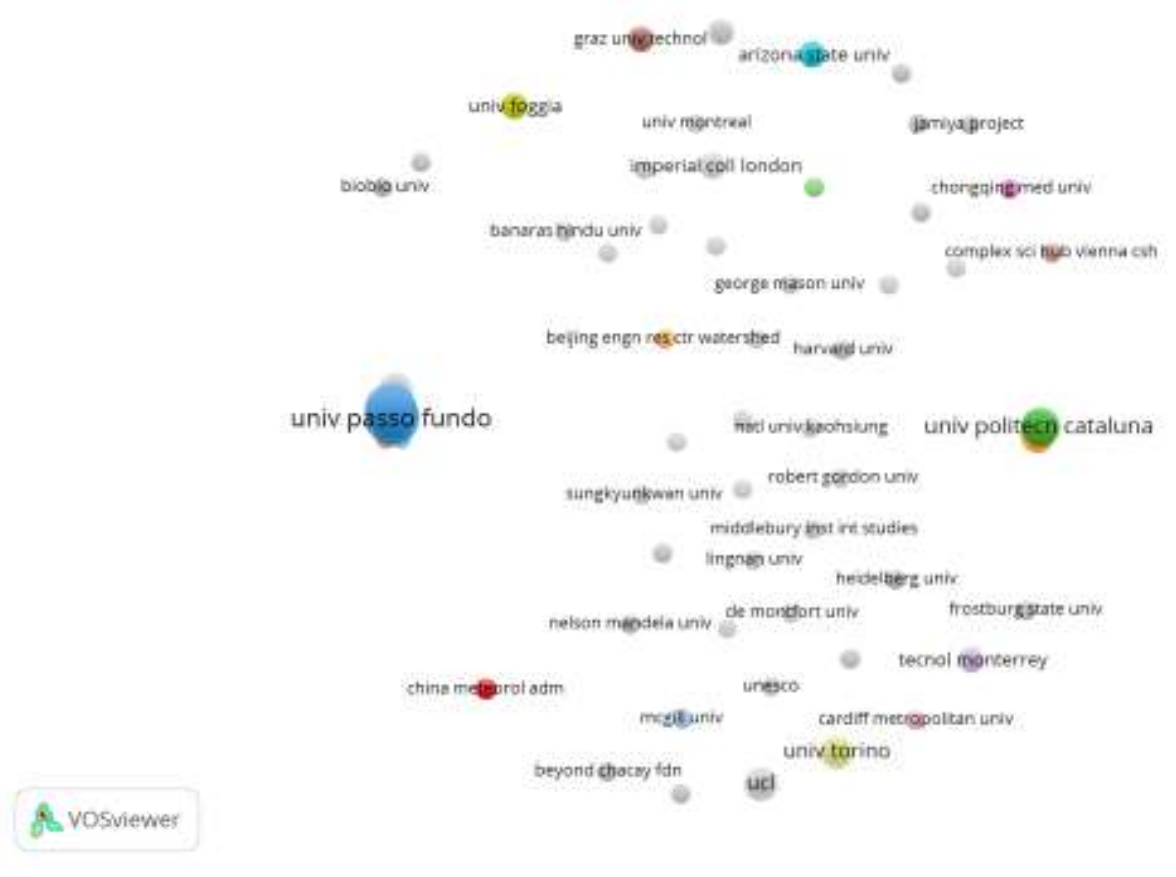

Fonte: VOSviewer (2021).

Foram identificadas 174 organizações com publicações relacionadas ao tema ODS e universidades, com um total de 357 conexões entre estas. Observa-se, no entanto, que a maioria destas não figura em nenhum cluster. Isso se justifica pela natureza recente das publicações sobre $\mathrm{o}$ assunto.

A partir da análise dos clusters, evidenciou-se a formação de 57. O mais significativo é o cluster 3 (em verde escuro, na figura 3), composto por 11 organizações. A Universidade Manchester Metropolitan é a que mais se destaca, com 19 publicações e uma relação de força tamanho 27, seguida da Universidade de Hamburger, com 16 publicações e uma relação de 
força tamanho 25 e da Universidade de Passo Fundo, com 16 publicações e relação de força 24. As demais universidades que compõem o cluster apresentam uma relação de força inferior ou igual a 5. Vale destacar que, neste cluster, encontram-se três universidades brasileiras, a saber: a Universidade de Passo Fundo, a Federal de Santa Maria e a Universidade Federal do Pampa. Esta apresenta apenas 2 publicações e relação de força tamanho 2. A figura 3 apresenta a relação entre as organizações que compõem o cluster.

Figura 3. Rede de co-autoria, por organizações - Cluster 3.

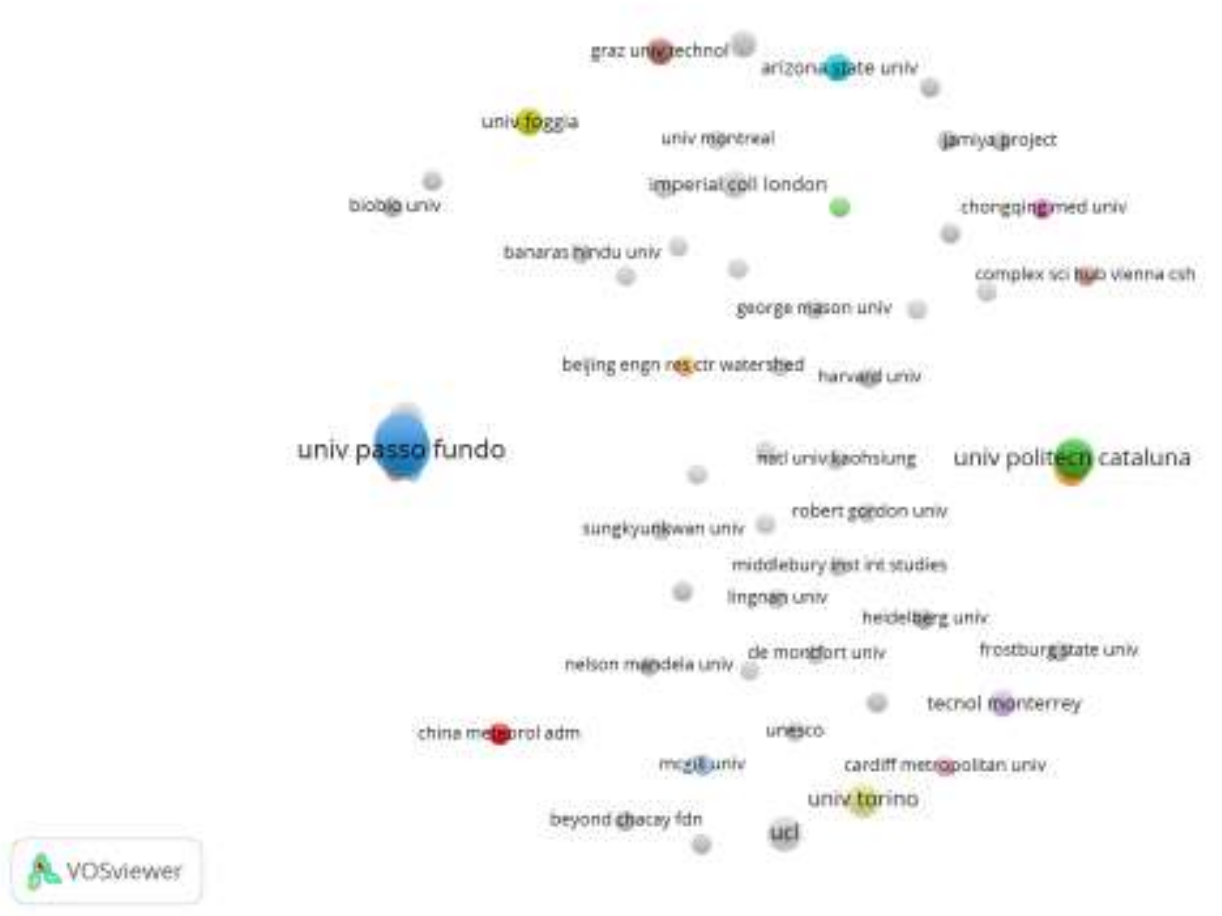

Fonte: VOSviewer (2021).

A Figura 4 apresenta o total da relação de força que as organizações mantêm entre si. Vale destacar que a Universidade de Passo Fundo figura entre as três que detêm um total de força maior, com tamanho 27, como pode ser observado na figura. 
Research, Society and Development, v. 10, n. 9, e12710917863, 2021

(CC BY 4.0) | ISSN 2525-3409 | DOI: http://dx.doi.org/10.33448/rsd-v10i9.17863

Figura 4. Total da relação de força, por organizações.

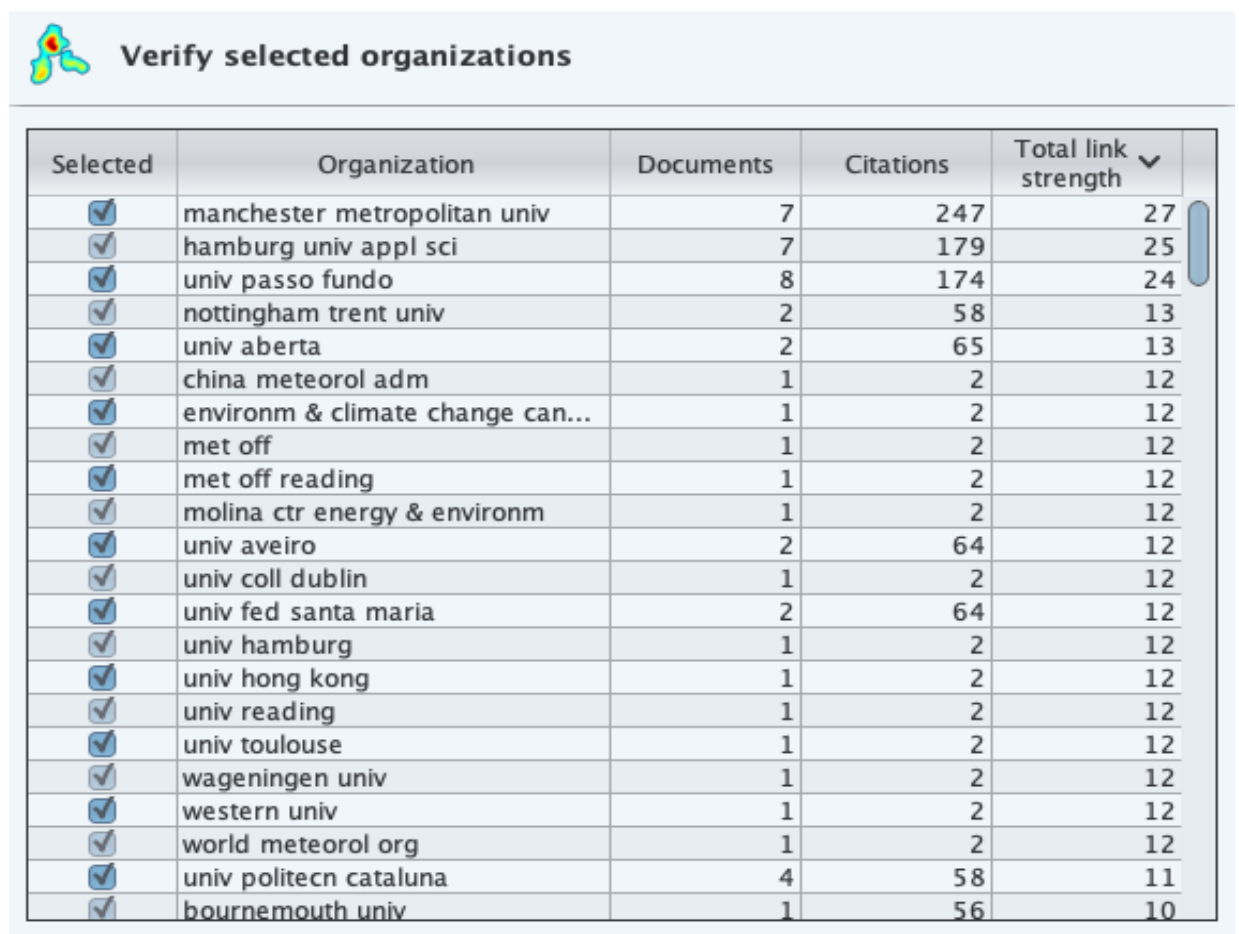

Fonte: Web of Science (2021).

A Figura 5, a seguir, mostra a formação de clusters por países. Observa-se que são poucos países que se encontram isolados e sem participação efetiva nos 12 principais clusters.

Figura 5. Rede de co-autoria por países.

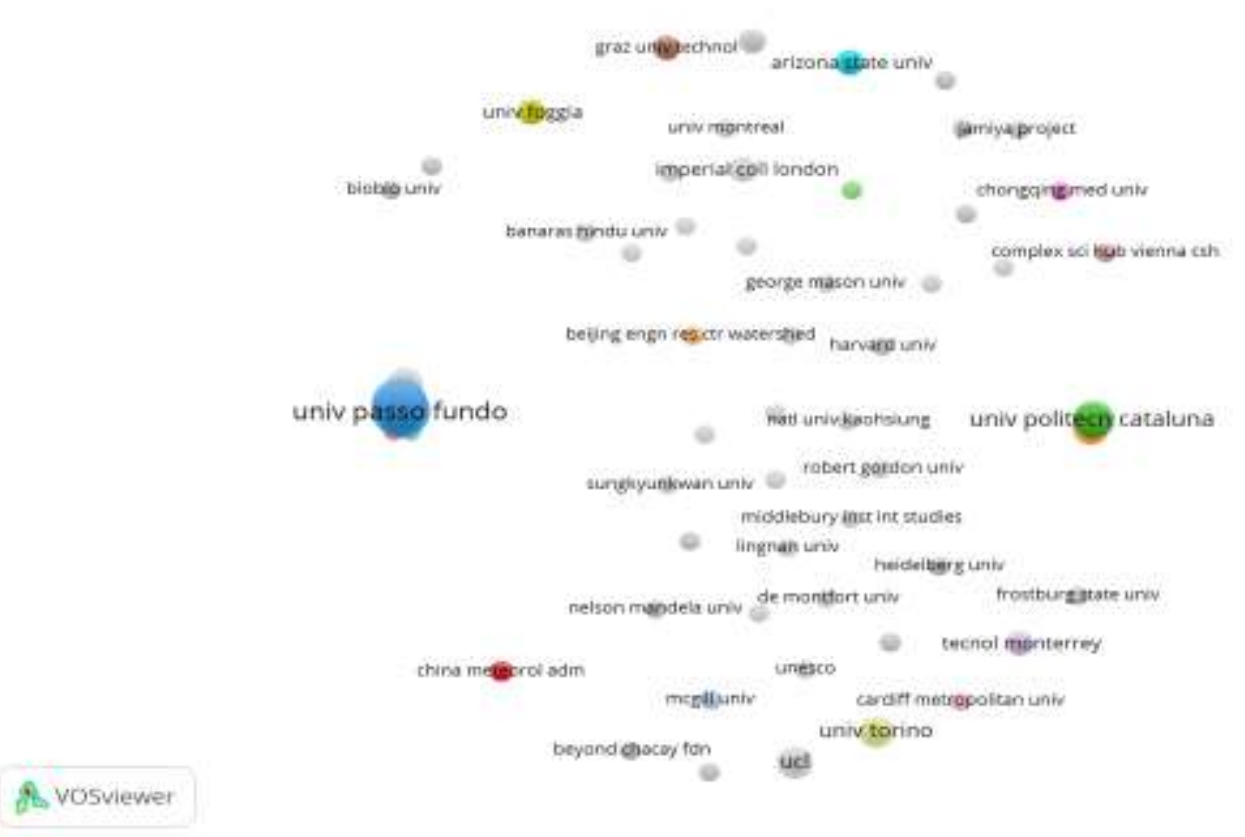

Fonte: VOSviewer (2021).

Constatou-se a participação de 37 países com publicações relacionadas ao tema em estudo e com 126 conexões entre si. Nesse sentido, destacam-se 5 países, com relações de força e quantidade de publicações, respectivamente: Inglaterra (22 e 
39), Alemanha (12 e 32), Brasil (11 e 20), Espanha (20 e 13) e Estados Unidos (11 e 19). Isso significa que, nesses países, há um grande interesse, entre os autores, em pesquisar os temas ora analisados.

Essas publicações formam 12 clusters, sendo que, a Áustria, Coreia do Sul e Taiwan não estão conectados na rede, e não apresentam nenhuma relação de força com os outros países.

O principal cluster (na cor rosa) é formado por apenas 2 países, Inglaterra e Kazaquistão, sendo a Inglaterra o país com maior destaque, na rede, com 22 publicações e tamanho de força 39. O cluster 1 (na cor vermelha), é composto pelos países: França, Irlanda, Japão, Holanda, China, Suiça e Estados Unidos. Este, é o país com maior destaque no cluster, com 11 publicações e uma relação de força tamanho 19, seguida da China, com 6 publicações e uma relação de força tamanho 12.

O segundo cluster com mais destaque é o 4 (na cor mostarda), formado por 4 países: Alemanha, India, Malta e Portugal, sendo que a Alemanha se destaca, com 12 publicações e uma relação de força tamanho 32.

O Brasil se encontra no cluster 7 (na cor laranja na Figura 5), com 11 documentos publicados e uma relação de força tamanho 20. As relações do Brasil dentro do cluster são apresentadas na Figura 6:

Figura 6. Rede de co-autoria, Brasil.

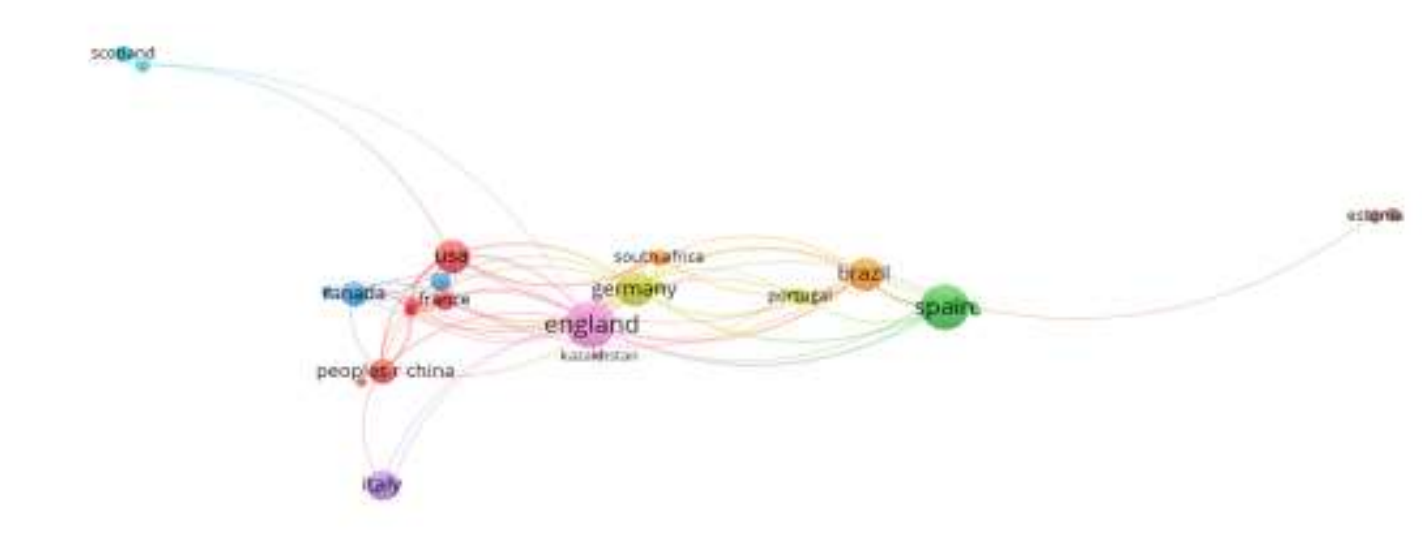

Fonte: VOSviewer (2021).

Conforme se observa, o Brasil mantém relação com Portugal, Espanha, Estônia, África do Sul, Alemanha, Inglaterra, EUA, França, Canadá e China, sendo a relação mais forte com a Alemanha e Inglaterra (linha de conexão mais densa).

Quanto aos temas explorados, para mapear campos de estudo e temáticas das publicações sobre os ODS e as universidades, elaborou-se a rede de co-ocorrências de termos, apresentados na Figura 7. 
Figura 7. Rede de co-ocorrência de termos.

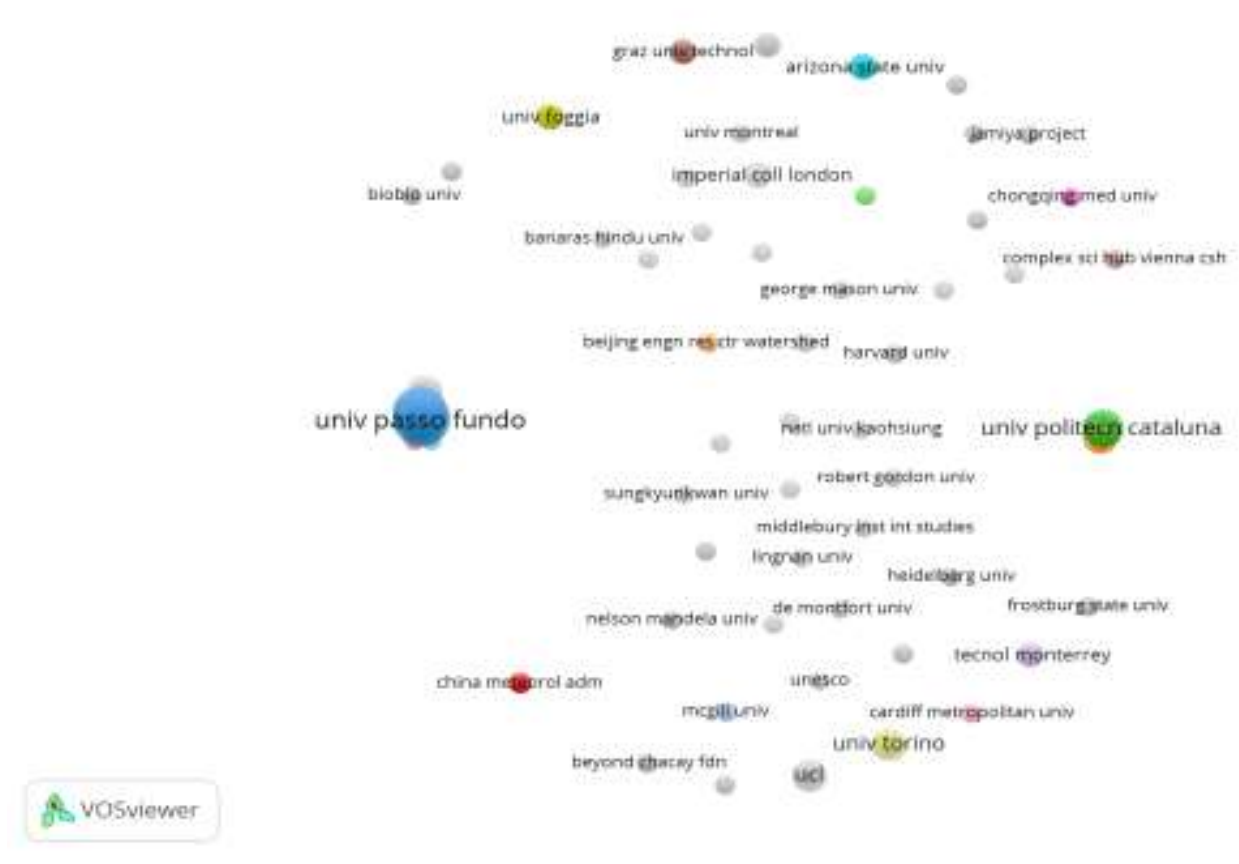

Fonte: VOSviewer (2021).

Dentre os 518 termos encontrados e suas 3981 conexões, os 08 termos de maior destaque e suas relações de força são, respectivamente: Objetivos do Desenvolvimento Sustentável (310), Ensino Superior (196), Desenvolvimento sustentável (183), Sustentabilidade (163), Universidades (141), Ensino-superior (145), Educação (116) e Educação para o desenvolvimento sustentável (95). Com isso, foram identificados 30 clusters, sendo 3 de maior destaque com 99 termos.

No primeiro cluster, com 36 termos (em cinza, na Figura 7) destaca-se o termo "objetivos do desenvolvimento sustentável" (relação de força tamanho 56). O segundo cluster, com 32 termos (em verde na Figura 6) destaca o termo "sustentabilidade" (relação de força tamanho 163). Este apresenta forte relação com os termos "universidades", "educação superior", “objetivos do desenvolvimento dustentável” e "alterações climáticas”.

Vale destacar que a primeira palavra-chave selecionada para este estudo, “objetivos do desenvolvimento sustentável”, encontra-se nos clusters 1, 14, 20 e 26, sendo que, neste último, apresenta uma relação de força mais significativa (tamanho 310). Já a segunda palavra-chave, "universidades", encontra-se nos clusters 2 e 21, com uma relação de força maior (tamanho 141), neste último cluster.

\subsection{Principais publicações e rede de citações}

As principais publicações, considerando o período escolhido para esta pesquisa (2016 - 2020), pertencem aos autores: Leal Filho, Brandli, \& Salvia.

A Figura 8 mostra a rede geral de citações de publicações, a partir de 2016. 
Figura 8. Rede geral de citações.

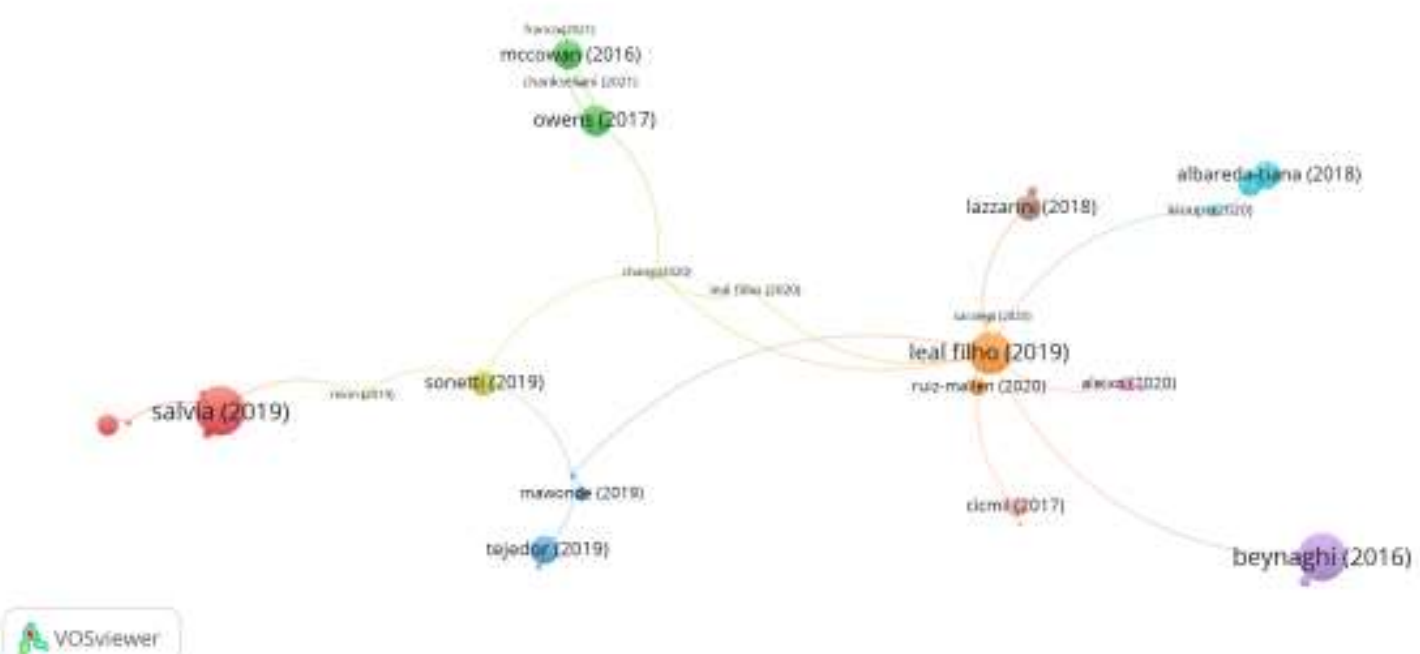

Fonte: VOSviewer (2021).

Com base nos resultados apresentados, a partir da criação dos mapas bibliométricos, gerados na Web of Science, verificou-se uma soma de 688 citações, resultando numa média de 7,82 citações, por ano, conforme se observa na Figura 9, que segue.

Figura 9: número de citações.

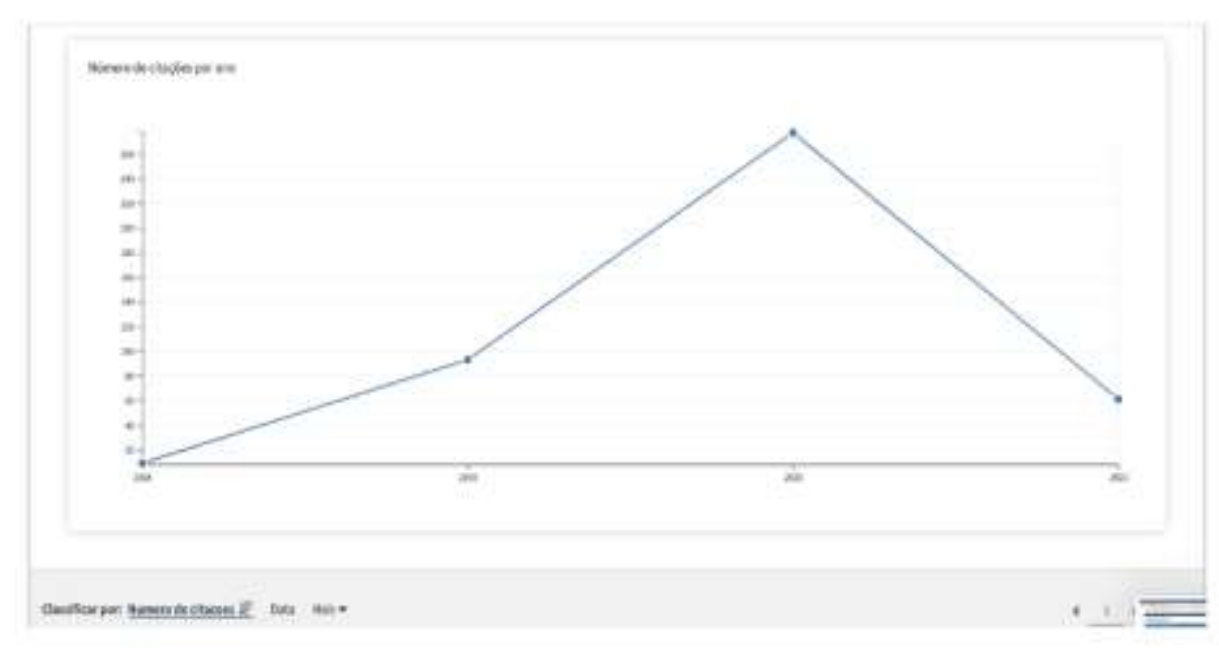

Fonte: Web of Science (2021).

À vista disso, observa-se que as publicações nessa área começaram a surgir a partir de 2016, tendo em vista a criação dos ODS, em 2015, por essa razão, optou-se pelo recorte temporal 2016 a 2020 para realização desta pesquisa, sendo que, as publicações relacionadas aos dois temas em estudo, começaram a surgir em 2016, e, de forma pouco significativa, foi aumentando nos anos subsequentes, porém, percebe-se que o número de publicações começa a crescer, e nos anos 2019 e 2020, houve um crescimento exponencial das publicações anuais. Observa-se, ainda, que, em 2021, foram publicados dois 
trabalhos, os quais não iremos discutir aqui, uma vez que a data não está no escopo desta pesquisa.

A publicação que recebeu maior score de citações foi: Sustainable Development Goals and sustainability teaching at universities: Falling behind or getting ahead of the pack? de Leal Filho, W. (2019), que consiste em uma pesquisa mundial implantada para coletar dados sobre os ODS e o ensino da sustentabilidade nas universidades.

A ideia do autor é mostrar que o engajamento da comunidade mundial, visando alcançar os Objetivos de Desenvolvimento Sustentável, significa que uma oportunidade inigualável é fornecida às universidades, tanto no que diz respeito ao ensino e à pesquisa, quanto aos ODS individuais, bem como na prossecução da sua "terceira missão" articulação com stakeholders externos e com a sociedade. No entanto, para ele, poucas universidades perceberam isso e muitas estão ficando para trás.

Nesse sentido, explora as muitas vantagens da introdução dos ODS no ensino e sugere que pode catalisar o engajamento de alunos em Instituições de Ensino Superior (IES) com os conceitos de sustentabilidade. O artigo preenche uma lacuna de pesquisa, levantando o estado da arte atual sobre o tema, apresentando dados atuais que descrevem até que ponto as IES estão usando os ODS para apoiar seu trabalho de sustentabilidade. Além disso, o autor mostra as razões pelas quais algumas instituições atualmente não estão engajadas.

Destaca-se a importância dessa pesquisa por ser a única no sentido de que oferece, pela primeira vez, uma visão geral do nível de ênfase que as universidades selecionadas atualmente colocam nos ODS. Além de fornecer uma contribuição para o estado atual do conhecimento ao delinear algumas ações que as universidades podem tomar para avançar com sua implementação.

A Figura 10 apresenta a rede de citações, a partir do referido autor.

Figura 10. Rede de citações, tendo Leal Filho como base.

Fonte: VOSviewer (2021).

Conforme se observa, a publicação de Leal Filho, em 2019, teve impacto e fundamentou uma rede composta por 08 publicações e 66 links de citações entre si.

A segunda obra analisada foi: Assessing research trends related to Sustainable Development Goals: local and global 
issues, de Salvia.

Figura 11. Rede de citações, tendo Salvia A.L. como base.

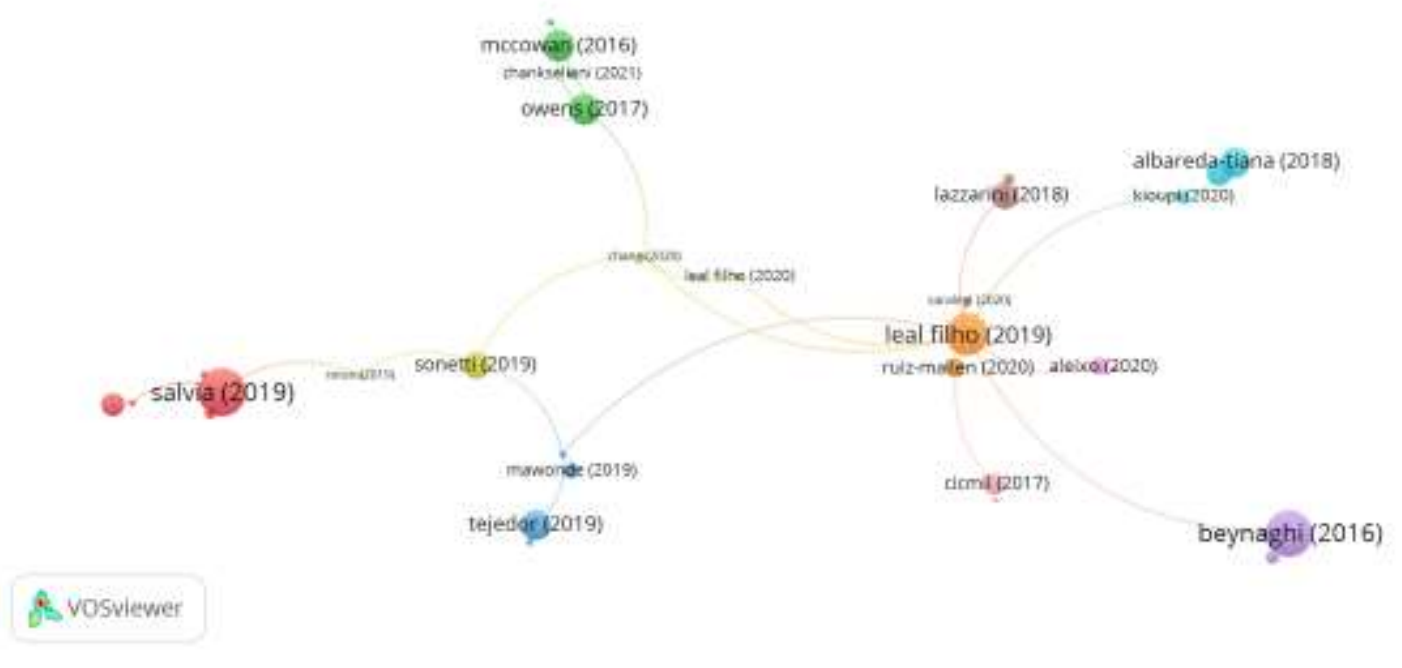

Fonte: VOSviewer (2021).

O objetivo da autora foi identificar os principais Objetivos de Desenvolvimento Sustentável abordados por especialistas de diferentes regiões geográficas, de acordo com sua experiência e área de pesquisa, e discutir a relação entre esses objetivos e as principais questões e desafios locais de cada região.

Esta pesquisa ressalta a existência de uma relação entre os problemas ou desafios locais observados em algumas regiões e as principais áreas de interesse dos especialistas pesquisados. Nela, a autora apresenta um conjunto de exemplos de situações positivas e negativas nas várias regiões, e considera até que ponto alguns Objetivos de Desenvolvimento Sustentável estão sendo perseguidos por investigação a nível mundial.

Nesse sentido, destaca-se a necessidade de um maior aprofundamento para se ter mais informações acerca do tema estudado. Ao mesmo tempo em que se reconhece a importância da base de dados para análise das publicações consideradas de impacto no campo de estudo delimitado, o que facilitou o detalhamento do estudo, bem como as características das publicações científicas da área.

\section{Considerações Finais}

Este estudo teve como objetivo analisar a produção científica internacional das universidades, em torno dos ODS, tendo o período de 2016 a 2020 como recorte temporal escolhido para a pesquisa, em virtude de os Objetivos do Desenvolvimento Sustentável terem sido criados em 2015 pela Organização das Nações Unidas, através da nova agenda de desenvolvimento sustentável - Agenda 2030.

A pesquisa teve início na base de dados Web of Science, da Capes e, através do software VosViewer, construiu-se os mapas bibliométricos, aqui apresentados.

Nesse sentido, chegamos aos principais clusters de co-autoria, entre autores, países e organizações, além dos termos em evidência nas publicações sobre a temática.

Os dados também, permitiram-nos elaborar a rede de citações da temática estudada, assim como evidenciar as publicações de impacto no campo, tomando por base o Fator $\mathrm{H}$ ( $h$-index). 
A partir da análise das obras indexadas na base da WoS, em torno do tema estudado, observa-se que ainda há um longo caminho a se percorrer, em relação à implementação de políticas ativas, no interior das universidades, para o alcance dos ODS.

As obras analisadas nesta pesquisa apontam para uma necessidade maior da participação das instituições de ensino superior, pois estas atuam na formação de profissionais das mais diversas áreas de conhecimento, podendo influenciar de maneira decisiva as gerações atuais e futuras na formação de uma educação baseada nos princípios da sustentabilidade. Por outro lado, reconhecem que, alguns países, têm demonstrado preocupações em torno da criação de políticas eficazes, no sentido de contribuir com o alcance do desenvolvimento sustentável.

Em relação às obras mais citadas, sobre esta temática, percebe-se que a maioria está focada na abordagem quantitativa, objetivando, por exemplo, a criação de indicadores que possam servir de base para novas ações que possam ser desenvolvidas por parte das universidades e de seus stakeholders.

Por fim, com base nos resultados aqui evidenciados, sugere-se, para trabalhos futuros, continuar aprofundando o conhecimento da produção científica desenvolvida nas instituições de ensino, além das universidades, para conhecer as propostas mundiais para o alcance dos ODS. Além disso, reforçamos a importância de se utilizar outras bases de busca como Scopus, Google Scholar, Pubmed etc, visando complementar o estudo para que a produção científica na área objeto deste estudo seja considerada e os resultados possam direcionar à criação de políticas educacionais direcionadas ao desenvolvimento sustentável e, consequentemente, ao bem-estar da população.

\section{Referências}

Annan-Diabab, F.; Molinarib, C. (2017). Interdisciplinarity: Practical approach to advancing education for sustainability and for the Sustainable Development Goals. The International Journal of Management Education. 15, 73-83. https://doi.org/10.1016/j.ijme.2017.03.006

Araújo, C. A. (2006). Bibliometria: Evolução Histórica e Questões Atuais. Em Questão, 12, 11- 32.

BANCO MUNDIAL. Relatório Anual 2017. https://openknowledge.worldbank.org/.

Beynaghi A, Trencher G, Moztarzadeh F et al. (2016). Cenários futuros de sustentabilidade para as universidades: ir além da Década das Nações Unidas para o Desenvolvimento Sustentável. Revista de Produção Mais Limpa, 112, 3464-3478. https://doi.org/10.1016/j.jclepro.2015.10.117.

Comissão Nacional Para Os Objetivos De Desenvolvimento Sustentável (CNODS): Plano de Ação 2017-2019. CNODS, 2017. https://bit.ly/2QNUCCp.

De la Poza, E.; Merello, P.; Barberá, A. \& Celani, A. (2021). Universities’ Reporting on SDGs: Using THE Impact Rankings to Model and Measure Their Contribution to Sustainability. Sustainability, 13, 2038. https://doi.org/10.3390/ su13042038

Dlouhá, Jana \& Pospíšilová Dlouhá, Marie (2017). Education for Sustainable Development Goals in public debate: The importance of participatory research in reflecting and supporting the consultation process in developing a vision for Czech education. Journal of Cleaner Production 172 . doi:10.1016/j.jclepro.2017.06.145

Dzimińska, M. et al. (2020). A Conceptual Model Proposal: Universities as Culture Change Agents for Sustainable Development. Sustainability, 12, 4635; $10.3390 /$ su12114635.

Hák, T., Janoušková, S., \& Moldan, B., Sustainable development goals: A need for relevant indicators. Ecological Indicators, 60(1), 565-573. 2016. doi:10.1016/j. ecolind.2015.08.003.

IPEA - Instituto De Pesquisa Econômica Aplicada. Agenda 2030: ODS - metas nacionais dos objetivos de desenvolvimento sustentável: proposta de adequação. Brasília: Ipea, 2018.

Kestin, T.; Van den Belt, M.; Denby, L.; Ross, K. E.; Thwaites, J.; \& Hawkes, M. (2017). Getting Started with the ODS in Universities: A Guide for Universities, Higher Education Institutions and the Academic Sector. https://resources.unsdsn.

Leal Filho, W., Brandli L. L., Becker, D., et al. (2018). Políticas de desenvolvimento sustentável como indicadores e pré-condições para os esforços de manutenção das universidades: fato ou ficção? International Journal of Sustainability in Higher Education 19(1): 85-113, https://doi.org/10.1108/IJSHE012017-0002.

Mayor, F. (1998). Preparar um futuro viável: ensino superior e desenvolvimento sustentável. In: Conferência mundial sobre o ensino superior. Tendências de educação superior para o século XXI. Anais da Conferência Mundial do Ensino Superior. Paris.

Neary, J., \& Osborne, M. (2018). University engagement in achieving sustainable development goals: A synthesis of case studies from the SUEUAA study. Australian Journal of Adult Learning, 58(3), 336-364.

Nilsson, M., Griggs, D., \& Visbeck, M. (2016). Policy: map the interactions between Sustainable Development Goals. Nature News, 534(7607), 320. 
Research, Society and Development, v. 10, n. 9, e12710917863, 2021

(CC BY 4.0) | ISSN 2525-3409 | DOI: http://dx.doi.org/10.33448/rsd-v10i9.17863

Objetivos De Desenvolvimento Sustentável: 17 objetivos para transformar o nosso mundo. https://www.piscodeluz.org/desenvolvimento-sustentavel.

Rede De Soluções Para O Desenvolvimento Sustentável - SDSN BRASIL (2015). https://www.conservation.org.

Roma, J. C. (2019). Os objetivos de desenvolvimento do milênio e sua transição para os objetivos de desenvolvimento sustentável. Cienc. Cult. 71 http://dx.doi.org/10.21800/2317-66602019000100011

Rosen, M. A. (2019). As universidades contribuem para o desenvolvimento sustentável? Eur. J. Sustain. Res. 4, 01-12.

SSDN. (2013). SDSN Thematic Group on Sustainable Cities. Supported by UN-Habitat, UCLG, Cities Alliance, and ICLEI. Why the world needs an urban development goal. https://sustainabledevelopment.un.org/content/documents/2569130918-SDSN-Why-the-World-Needs-an-Urban-SDG.pdf.

Umar, T. (2020). Uma abordagem integrada de gestão da sustentabilidade para as universidades. Processo da Instituição de Engenheiros Civis Sustentabilidade da Engenharia 173(7): 344-355, https://doi.org/10.1680/jensu.19.00014

UNESCO. (2017). Unesco avança na Agenda 2030 para o Desenvolvimento Sustentável. UNESCO: https://sustainabledevelopment.un.org/conteúdo/documentos/21252030\%20Agenda\% 20\%20Sustainable\%20Development\%20web.pdf.

United Nations. (2015). Transforming Our World: The 2030 Agenda for Sustainable Development. UN General Assembly, www.refworld.org/docid/57b6e3e44.html.

Van Eck, N. J., \& Waltman, L. (2017). Citation-based clustering of publications using CitNetExplorer and VOSviewer. Scientometrics, 111, 1053-1070. 